For Internal Distribution Only

Accelerator Division

Alternating Gradient Synchrotron Department BROOKHAVEN NATIONAL LABORATORY

Upton, New York 11973

Accelerator Division

Technical Note

AGS/AD/Tech. Note. No. 428

BEAM LOSS ISSUES FOR AGS HIGH INTENSITY HEP

S. Y.Zhang

March 5, 1996 


\title{
BEAM LOSS ISSUES FOR AGS HIGH INTENSITY HEP
}

\author{
S. Y. Zhang
}

\section{Introduction}

After the beam entered the AGS ring, most beam losses have happened in the injection porch, the RF capture, and in a vicinity of transition. In this note, we study these losses separately with respect to the longitudinal and the transverse effects. The following issues are relevant to the understanding and the dealing with the beam loss at high intensity.

1. The longitudinal emittance needs to be monitored, and used in the study of the beam loss.

2. The performance of each batch needs to be learned, based on their emittances.

3. A better knowledge of the broad band impedance is needed.

4. The transverse emittance also needs more attention at the higher beam intensity.

\section{BTA Transfer}

\subsection{Longitudinal}

The longitudinal quadrupole mode can be excited at the Booster before the extraction, while the Booster $\mathrm{RF}$ voltage can be from $60 \mathrm{KV}$ to $80 \mathrm{KV}$ before the extraction. If the quadrupole mode is excited, then the bunches are extracted when longest. The beam is received at the AGS with or without phase mismatch, i.e. with or without dipole oscillation. The VHF is on for about $60 \mathrm{~ms}$ for each transfer and the RF voltage can be from $60 \mathrm{KV}$ to $200 \mathrm{KV}$, and most likely to be at $100 K V$ or a little higher.

We consider the function of the VHF as painting the longitudinal phase space, according to the bucket and the largest extent of the bunches in either length or height. The longitudinal emittance is defined according to both the bucket and bunch.

The bucket lengths are the same for the Booster and the AGS, therefore, we only need to know the bucket height, which for convenience we use the maximum momentum deviation to represent,

$$
\left(\frac{\Delta p}{p}\right)_{B K}= \pm \sqrt{\frac{2 e V_{r f}}{\pi h \beta^{2} E|\eta|}}
$$

The bucket heights are as follows, 


\begin{tabular}{|c|c|c|c|c|c|c|}
\hline & $h$ & $\beta$ & $E$ & $\eta$ & $V_{r f}$ & $(\Delta p / p)_{B K}$ \\
\hline Booster & 2 & 0.926 & $2.49 \mathrm{GeV}$ & -0.099 & $60 \sim 80 \mathrm{KV}$ & $0.95 \% \sim 1.1 \%$ \\
\hline AGS & 8 & 0.926 & $2.49 \mathrm{GeV}$ & -0.128 & $60 \sim 200 \mathrm{KV}$ & $0.42 \% \sim 0.76 \%$ \\
\hline
\end{tabular}

Table 1

If the buckets are matched, then the quadrupole oscillation excited at the Booster continues at the AGS, which is damped later by the VHF. From Table 1, we see that the bucket height at the AGS is always smaller than the one at the Booster and the quadrupole oscillation will be weakened at the AGS. As an extreme example, let the RF voltage at the Booster be $60 \mathrm{KV}$ and also the bunch length be enlarged by $50 \%$ using the quadrupole oscillation before the extraction, then a $138 K V \mathrm{RF}$ voltage at the AGS will cease the quadrupole oscillation completely at the AGS, owing to the match of the bunch to the bucket. With the AGS RF voltage smaller than $138 K V$ the quadrupole oscillation will continue, however, the particles with large momentum deviation in the bunch, rather than phase deviation, will be responsible for the longitudinal emittance blow-up. In other words, without the quadrupole oscillation excited at the Booster, a larger longitudinal emittance will be defined. It is also true that under this condition the bunch will be shorter as arriving the AGS and the beam peak current will be larger, which implies a larger 'slow' loss due to the space charge effect.

With the dipole motion, excited by the phase mismatch, a larger longitudinal emittance blow-up can be obtained, compared with the pure quadrupole oscillation. It was observed that with dipole motion, the dilution process was not completed within the $60 \mathrm{~ms}$ VHF duty period, which causes a further blow-up at the following VHF duty period. This definitely poses an 'unfair' condition for the 4 th transfer.

It would be of interest to learn more about the VHF dilution, where a given longitudinal emittance blow-up should be used as the criterion. Note that with or without quadrupole oscillation at the Booster, with or without phase mismatch at the AGS, the choices of the Booster and the AGS RF voltage, the VHF duty period and its tune, these are all relevant to the longitudinal emittance blow-up.

Once the longitudinal emittance has been defined, it will determine the beam loss at the accumulation, the RF capture and the transition. . At the lower intensity, almost all measures have been toward to larger emittance. At the higher intensity, some attempt have been observed to limit the longitudinal emittance blow-up, for example, to not use the phase mismatch and to raise the AGS RF voltage on the injection porch. The better knowledge of the beam longitudinal emittance, and probably even the emittances of each batch, is needed in understanding the beam loss in the rest part of the cycle.

\subsection{Transverse}

The transverse emittance can be defined, to a lesser extent, by changing the Booster tune and adjusting the AGS injection bumps, etc., which, meanwhile, also affect the transfer efficiency. So far the adjustment relevant to the transverse emittance has been judged mainly by improving the transfer efficiency. Therefore, the transverse emittance is not as controllable as its longitudinal counterpart. As the intensity gets higher, smaller transverse beam size might be of interest, and this scenario could change. 


\section{Accumulation Loss}

\subsection{Longitudinal}

The longitudinal microwave instability is not a serious problem. Taking the AGS RF voltage $V_{r f}=150 \mathrm{KV}$, and the bunching factor $B=0.5$, assuming that the bunches have already been diluted, we calculate that the bunch momentum spread as,

$$
\left(\frac{\Delta p}{p}\right)_{B H}=\sqrt{\frac{\pi e V_{r f}}{2 h \beta^{2} E|\eta|}} B\left(1-\frac{B^{2}}{2.5}\right)=0.47 \%
$$

where $1-B^{2} / 2.5$ is the correction factor for the RF bucket nonlinearity. For the normalized transverse emittance $\epsilon_{N, 95 \%}=100 \pi \mu m$, at the injection the longitudinal space charge impedance is $Z_{L S C} / n=-j 102 \Omega$. Taking the broad band impedance $Z_{L B B} / n=j 30 \Omega$, we have the total impedance $Z_{L} / n=-j 72 \Omega$. The average beam current at $N=6 \times 10^{13}$ particles is $I_{0}=3.3 \mathrm{~A}$, and the peak current is $I_{\text {peak }}=2 I_{0} \sqrt{2 / \pi} / B=10.53 \mathrm{~A}$. Therefore the momentum spread threshold is,

$$
\left(\frac{\Delta p}{p}\right)_{T H S D}=\sqrt{\frac{e I_{\text {peak }}}{\beta^{2} E|\eta|}\left|\frac{Z_{L}}{n}\right|}=0.17 \%
$$

which is smaller than the beam momentum spread, and the beam is, therefore, stable. We note, however, the stability margin will be smaller as the bunches just arrive at the AGS. For instance, it is ready to calculate that if the bunch matches the bucket with the bunching factor $B=0.25$, then the beam momentum spread is reduced to $0.25 \%$, meanwhile, the peak current becomes $21.1 A$, and the momentum threshold is increased to $0.24 \%$, about the same as the beam momentum spread.

We note that if the bunch arrives at the AGS with the longer bunch length, then the beam peak current will be smaller, and also the beam momentum spread is smaller. Since the contribution of the smaller beam peak current is overwhelmed by the reducing of the momentum spread, the longitudinal microwave instability becomes more likely to happen.

For the beam survival at the accumulation, however, the longer bunches are much needed, which implies a smaller peak current and hence a smaller incoherent tune spread. In general, given an RF voltage, i.e. a bucket height, a longer bunch agrees with a larger longitudinal emittance. A larger longitudinal emittance, however, also implies a larger bunch momentum spread, which is favorable in terms of the microwave instabilities, but unfavorable in terms of the RF capture beam loss, and the transition loss. One way to handle this problem is to manipulate the longitudinal emittance, i.e. for a given emittance, to lower the RF voltage, which gives rise to a longer bunch, but with a smaller momentum spread. With the use of a lower RF voltage on the entire injection porch, two problems may arise as follows,

1. A lower RF voltage is not favorable to the longitudinal instabilities. We have seen that even for the fairly high AGS injection RF voltage of $V_{r f}=150 \mathrm{KV}$, the stability margin is not large in the early part of the injection.

2. The RF voltage also plays a major role in defining the longitudinal emittance, and this low voltage may not be desirable in this aspect.

Under the condition that the dilution is completed within the VHF $60 \mathrm{~ms}$ duty period, and an RF voltage adiabatic 'cut half' period of $10 \mathrm{~ms}$, it is not harmful to try to lower the 
RF voltage between the VHF duty period. By lowering the RF voltage to half, the bunch length can be increased by some $20 \%$.

\subsection{Transverse}

Taking the limiting transverse aperture of $b=35 \mathrm{~mm}$ at the amplitude function $\beta(s)=22$ $m$, the machine transverse acceptance is $A_{T}=b^{2} / \beta(s)=56 \pi \mu m$. The maximum allowed emittance can be $\epsilon_{N, 95 \%}=100 \pi \mu m$, which accounts for $\epsilon_{N, 95 \%} / A_{T} \beta \gamma=73 \%$ within the acceptance at the injection, $\beta \gamma=2.45$. This also implies a beam with $\sigma=12 \mathrm{~mm}$ at the injection, and $\sigma=6 \mathrm{~mm}$ at the transition with $\beta \gamma=9.4$. The large transverse emittance has two problems,

1. It meets the machine aperture.

2. The large emittance is unfavorable for the transverse microwave instabilities, which may arise about $300 \sim 500 \mathrm{~ms}$ after the transition.

The obvious advantage of the large emittance is that it implies a smaller incoherent tune spread, which so far is the most blamable for the 'slow' and 'drool' losses in the injection porch. It is not very clear that how much the 'emittance meets aperture loss' we have now. It is clear, however, that along with the progress on the stopband corrections and higher beam intensity, this problem will need some attentions.

The transverse microwave instability is believed not a problem at the low energy. The resistive wall coupled bunch instability is a serious problem, which, however, is being dealt with by the transverse damper quite well at the AGS. Both horizontal and vertical instabilities have been observed, and also an instability transferring from the horizontal to the vertical has been observed.

\section{RF Capture Loss}

\subsection{Longitudinal}

Consider a case that the RF voltage is $100 \mathrm{KV}$ on the injection and arises to $350 \mathrm{KV}$ in the acceleration, and let the bunching factor be $B=0.6$. A comparison of the bunch and moving bucket, for their length and height, shows no problem. The capture efficiency, however, is not perfect in the AGS. Taking a look at Fig.1, where the beam loss after the 4th transfer is an almost constant drool up to about $550 \mathrm{~ms}$. The magnetic field starts to ramp up from $480 \mathrm{~ms}$, therefore the drool, presumably due to the stopband crossing loss, should be flattened very rapidly, because that the incoherent tune spread would be reduced rapidly. The continue drool is, therefore, probably owing to the capture loss.

How to understand and deal with this problem?

1. Although a typical beam line density for protons is parabolic, Gaussian line densities seem more suitable for the AGS proton beams. This is probably owing to the VHF dilution we applied. With a Gaussian line density, the particles included in the bunch length $B=0.6$, which we measured for the portion of the bunch length within $\pm 2 \sigma$, are roughly $95 \%$. To include $99 \%$ particles, the relevant bunching factor becomes 0.76 , and $99.5 \%, 0.83$. It seems that as long as the VHF dilution is needed, one would have the Gaussian line density with long tails, and some losses are hard to eliminate. 
2. It is of more interest to know that which one is more responsible for the beam loss, the bunch length, or the height. This is a subject of the longitudinal emittance manipulation in the acceleration. A better knowledge of this aspect is desirable, since that with the increase of the intensity, the RF capture loss will be even harder to combat.

The bunching factor, the RF voltage, the $\dot{B}$, and VHF programs are shown in Fig.2 for the 4th transfer, which explains that the slow loss of the 4 th batch will be larger than others, because that the RF voltage starts ramping even during the dilution period. Although the total effect of this program in terms of the beam loss may be desirable, it is worth to track the beam loss well after the transition to see the performance of each batch, based on their emittances.

\subsection{Transverse}

Ideally the transverse issues are irrelevant to the RF capture loss. From Fig:2, which was taken during the 1995 SBE, we can find that the transverse loss is mixed with the longitudinal one. Before the magnetic field ramping, the RF voltage starts rising, which presumably is getting the bunches ready for the capture. Since the bunch length is shortened, however, the beam peak current arises, and the incoherent tune spread becomes larger. This causes additional loss, until the tune spread finally gets smaller at about $550 \mathrm{~ms}$. This makes the identification of the loss source very difficult.

\subsection{Discussion}

To improve the RF capture efficiency, we consider the following choices.

1. A better RF voltage program, if still possible, should be applied to fully utilize the capture capability.

2. A longer capture period and a smaller longitudinal emittance can improve the RF capture loss. These two choices are, however, prohibitive in terms of the accumulation loss.

3. To improve the VHF dilution for better particle distribution, i.e. to eliminate the long tails of the line density. The use of the dipole motion in the dilution seems not favorable at this point.

4. Second harmonic RF on the injection porch will be of interest, which implies both the lower peak current and short tails.

\section{Transition}

\subsection{Longitudinal}

It is known that the beam loss at the transition consists of a 'fast' one in a matter of a few $m s$ in the vicinity of the transition jump, and a 'slow' one in a matter of several tens of $m s$ below the transition, during the jump. The fast loss can be reduced by making the jump larger, which in turns deteriorates the slow loss. The fast loss is mainly owing to the classical reasons, which can be listed as, 
1. Because of the beam momentum spread, the transition gamma is distributed within the particles, therefore, the particles cannot agree on a same transition. This is the 'Johnsen effect'. Since the magnetic field is kept ramping, those particles not keeping with the bucket phase jump are likely left behind, and beam loss occurs.

2. For coasting beams, only pure capacitive impedance is allowed below the transition, and only inductive impedance is allowed above transition. The case of the space charge impedance, which is purely capacitive, above the transition is called the 'negative mass instabilities'. In this sense, this instability does not apply to the AGS, because at the transition the broad band impedance, which is inductive, is dominant. However, considering the beam instabilities caused by the resistive impedances and the broad band impedance below the transition, some loss is likely to happen.

3. The RF system encounters a heavy beam loading around the transition, and also there is a bunch to bucket mismatching after the transition. The $\dot{B}$ noise, or somewhat unsteady magnetic field ramping is also a problem.

The first two problems are greatly alleviated by the gamma jump, with the RF system keeps up with it.

Under the gamma jump, the first look we will take is a kind of 'global' approach, i.e. we look at the time period of about $50 \mathrm{~ms}$ below, and $10 \sim 20 \mathrm{~ms}$ above the transition, and pay less attention on the short period of jump. The bunch lengths with the $B=0.6$ at the injection porch are shown in Fig.3, for without gamma jump, with the jump, and jump with also the RF voltage drop. The gamma jump and the RF voltage program are also shown. These effects are very important, because one sees that with the jump, the bunches never get, with the exception of the $1 \sim 2 \mathrm{~ms}$ jump, close to the transition, and therefore the bunch length is much longer. With the RF voltage drop, the situation is further improved, and the bunch length is even longer.

Also important is the 'potential well effect', which is the usual name for the variation of the longitudinal focusing due to the wide band impedance. This effect, if we take only the first order approximation, can be represented by the incoherent synchrotron frequency shift as,

$$
\omega_{S, i n c}=\omega_{S 0}\left(1+\frac{h I_{0}}{6 B^{3} V_{r f} \cos \phi_{S}} \operatorname{Im}\left(\frac{Z_{L}}{n}\right)\right)
$$

where $\omega_{S 0}$ is the synchrotron oscillation frequency with zero intensity. Note that the incoherent frequency shift affects only the quadrupole oscillation, not the dipole one. Below the transition, for the space charge impedance we have $\operatorname{Im}\left(Z_{L S C} / n\right)<0$, therefore the incoherent synchrotron frequency decreases, which implies the defocusing and a bunch lengthening. Above the transition, for the broad band impedance $\operatorname{Im}\left(Z_{L B B} / n\right)>0$, but also $\cos \phi_{S}<0$, therefore also the defocusing and the bunch lengthening. The space charge impedance depends on both the beam transverse size, i.e. the transverse emittance, and, more heavily, the beam energy. Thus, when the particles are accelerated, it reduces rapidly. This impedance is capacitive. The wall broad band impedance is an important problem, because we still do not have a good knowledge about it for the AGS. This impedance is inductive. Taking $Z_{L B B} / n=j 30 \Omega$, and the normalized transverse emittance $\epsilon_{N, 95 \%}=100 \pi \mu m$, the wide band impedance of the AGS is shown in Fig.4, which indicates that at around $4 \mathrm{GeV}$, this 
impedance is minimum, owing to the cancellation of the space charge and the broad band impedances.

Given the RF voltage, the potential well effect is proportional to the beam line density, the wide band impedance, and more importantly, to the factor of $1 / B^{3}$. At the above mentioned wide band impedance and the full intensity, the quadrupole oscillation frequency shift, which is usually used to measure these impedances, only in a matter of $10 \mathrm{~Hz}$, or a little larger, yet it depends heavily on the particle distributions. The incoherent synchrotron oscillation frequency during the cycle is shown in Fig.5, comparing with the synchrotron frequency with zero intensity. The difference is not large, and therefore, one may intend to underestimate this effect. A better way to evaluate the effect probably is to observe through the 'effective' RF voltage, which is the combination of the external voltage and the potential well force. This voltage is defined as,

$$
V_{r f, e f f e c t}=\left(\frac{\omega_{S, i n c}}{\omega_{S O}}\right)^{2} V_{r f}
$$

Without the gamma jump, the applied RF voltage and the effective voltage are shown in Fig.6, which shows,

1. At about $600 \mathrm{~ms}$ from the AGS $T_{0}$, the wall inductive impedance becomes dominant. Below the transition, the effect of this impedance is focusing, leads to the effective voltage reaching $5 M V$, and above the transition, it is defocusing, leads to entirely loss of the focusing force. This says that without the gamma jump, the beam with high intensity of $N=6 \times 10^{13}$ is simply impossible to get across the transition at the AGS.

2. The effect is negligible on the front porch. Above the transition, if the VHF dilution is applied, the bunches become longer, and this effect is also trivial.

With the gamma jump, the effective RF voltage is plotted with two RF voltage programs, in Fig.7. The upper plot shows the ones without voltage drop at the transition. Right below the transition, the effective voltage is about $600 \mathrm{KV}$, which is much larger than the one we know. The lower plot shows the ones with the RF voltage drop, above the transition it drops to about $100 \mathrm{KV}$. Fig.7 explains three things,

1. The bunch length rapid build up right above the transition, as shown in Fig.8, which is measured during the SBE with the bunch intensity of $1.7 \times 10^{12}$. The VHF program is also plotted. Although the VHF is on above the transition, by itself the rapid bunch length increase cannot be explained. It can be explained, however, by the drop of the effective voltage, which is defocusing and helps the bunch lengthening.

2. The very unstable beam above the transition, not below the transition. Without the VHF, there will be severe beam loss above the transition, which implies instabilities, most likely the longitudinal one. The effective synchronous phase is plotted in Fig.9, which is large above the transition, implying the longitudinal unstable beam. To reduce this effect, the rising of the RF voltage above the transition now takes much shorter time than the one plotted. This in turn has, however, transferred the pressure to the RF system.

3. A strong quadrupole oscillation above the transition, which can be explained by the bunch to bucket mismatch. The bunch can be seen as not changing much during the 
short jump. Fig.7 shows that the bucket height is abruptly reduced to half, since the effective voltage drops from about $400 \mathrm{KV}$ to about $100 \mathrm{KV}$. Therefore, a strong quadrupole oscillation will be excited.

The first fact shows also that the at the transition the dominant wide band impedance is inductive. Since the space charge impedance at the transition is about $-j 10 \Omega$, hence it is reasonable to assume a wall broad band impedance larger than $j 10 \Omega$.

\subsection{Transverse}

The momentum spread is a dominant factor in the beam loss below the transition, during the transition gamma ramping up. Taking the largest dispersion function during the jump $D=8 \mathrm{~m}$, and the limiting chamber width $b_{H}=75 \mathrm{~mm}$, the allowed $r \mathrm{~ms}$ beam size within the $73 \%$ aperture limit is $\sigma_{\text {allowed }}=73 \% \times 75 / \sqrt{6} \approx 22 \mathrm{~mm}$, where the factor $\sqrt{6}$ comes from that the $95 \%$ particles are included. Using,

$$
\sigma_{\text {allowed }}^{2}=\sigma^{2}+D^{2}\left\langle\left(\frac{\Delta p}{p}\right)^{2}\right\rangle
$$

where $\left\langle(\Delta p / p)^{2}\right\rangle$ is the mean square value of the momentum spread. For the beam size without dispersion $\sigma=6 \mathrm{~mm}$, we calculate the allowed FWHM beam momentum spread as

$$
\left(\frac{\Delta p}{p}\right)_{F W H M}= \pm 2 \sqrt{\left\langle\left(\frac{\Delta p}{p}\right)^{2}\right\rangle}
$$

which is $\pm 0.53 \%$. Note that the fairly large beam size $\sigma=6 \mathrm{~mm}$ contributes less than $4 \%$, therefore, it is negligible.

The beam loss during the jump is somewhat linear, because that when the dispersion becomes larger, the beam momentum spread is reduced. The beam radial position shift may also contribute to this.

\subsection{Discussion}

It seems that with a large jump, the fast loss in a very vicinity of the transition is improved significantly. The slow loss has to be eventually reduced by the 'local distortion' or 'linear jump' system. Until this scheme is realized, one has to deal with the classical transition fast loss, using a smaller jump. This is our second look, which takes into account of the classical fast loss. One example is shown in Fig.10, where one sees that a smaller jump with 1.25 unit can bring the situation half way back to the non-jump era. Therefore, better understanding on the fast loss is of interest.

The mechanism of the fast loss is not completely understood. Conventionally, it is attributed to mainly two sources, one is the 'Johnsen effect', and another is the longitudinal microwave instabilities. If the magnetic field ramping at the transition can be stopped or much slowed, the loss owing to the Johnsen effect might be identifiable, but this is difficult. The remedy for the Johnsen effect is to lower the beam momentum spread, which we did, and also to use the sextupole correction, which we plan to do. On the other hand, the situation associated with the microwave instabilities are more complicated. Several related factors are discussed as follows. 
1. In a very vicinity of transition, the frequency slippage factor $\eta$ is very close to zero. The Landau damping is vanished. A comparison of the microwave instabilities for with and without jump is shown in Fig.11.

2. A longer bunch implies a lower peak current, however, the more important momentum spread is also brought down, and this is not favorable for the instabilities. The real reason why the longer bunches are still desired at the transition for this point is that the longer bunch agrees with larger $|\eta|$, i.e. to stay away from the transition. In this sense, whenever the jump is adjusted, the ramping $\gamma$ should be placed in the middle of the jump. An example is shown in Fig.12, where for the same size of jump, the one followed this rule looks in a better shape.

3. The observation shows that below the transition, the instability seems not to be a problem, the lower momentum spread is still much needed. We note that, however, once an instability occurs right below the transition, one can expect not just a minor beam loss, but a messy situation. Above the transition, the lower effective RF voltage has induced much crucial condition for the microwave instabilities. Using the rapidly increased RF voltage and the VHF, the momentum spread is brought up to combat the instabilities.

\section{Acknowledgment}

The very helpful discussions with $\mathrm{L}$. Ahrens, T. Roser, and K. Zeno are appreciated.

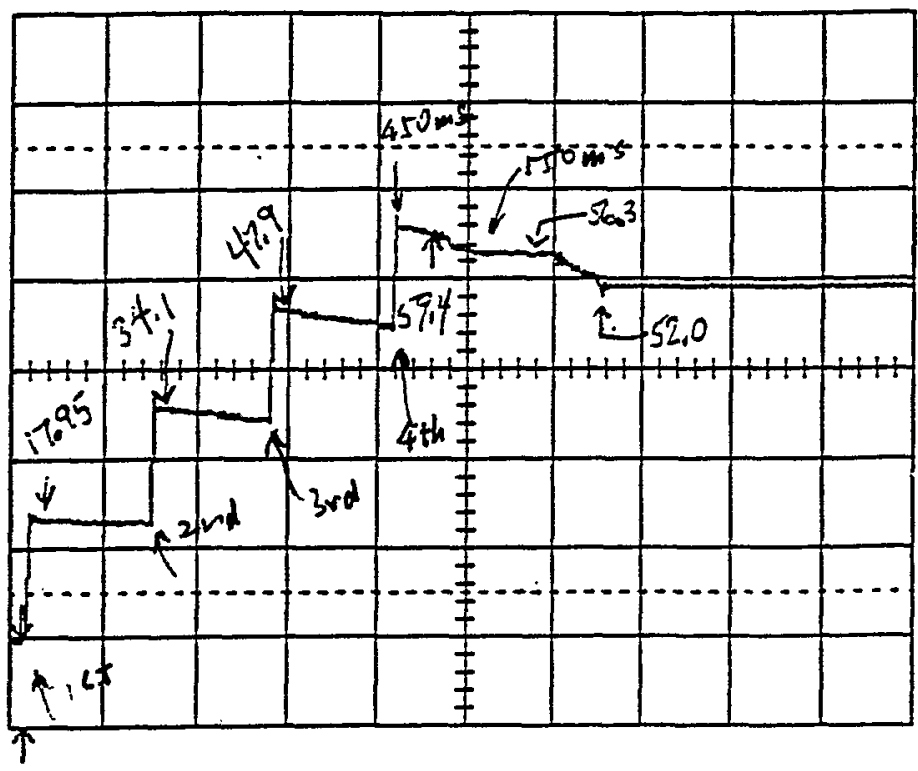

Fig.1. Beam Loss at the CBM $60 \mathrm{TP}$ 


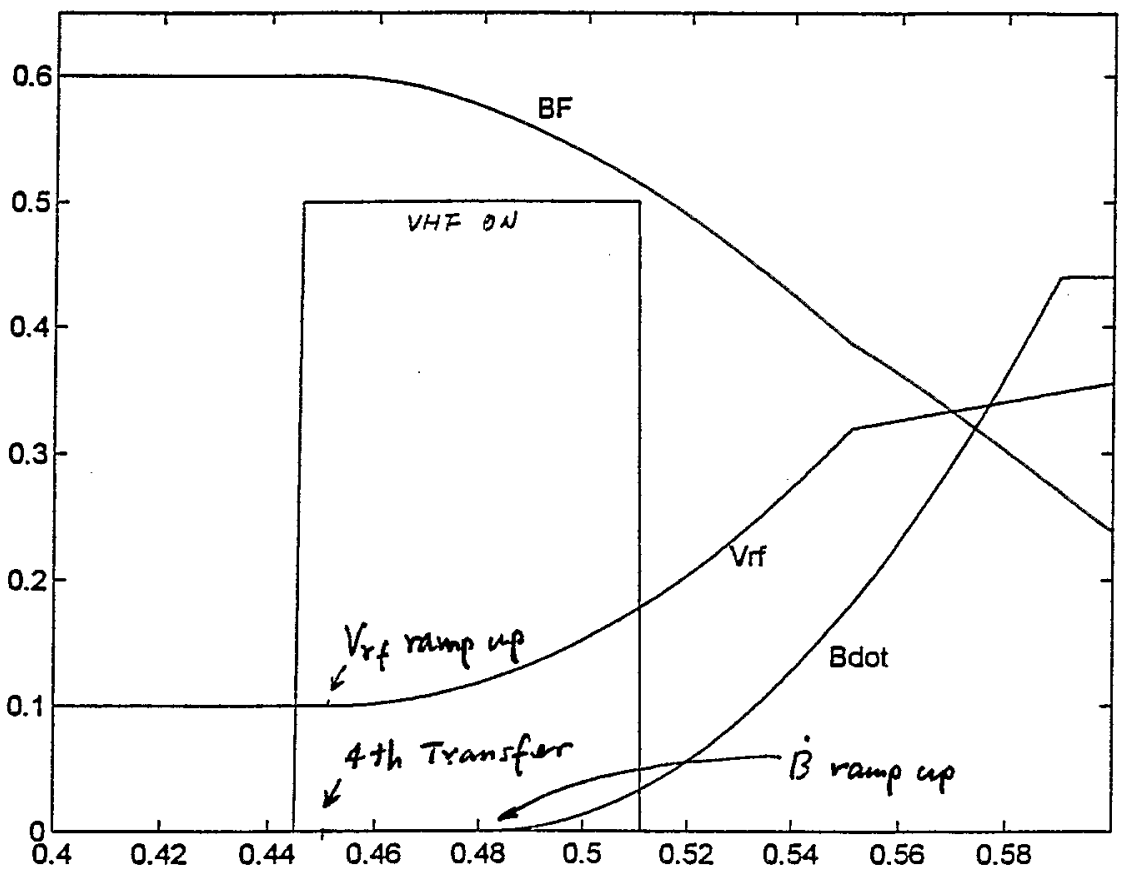

Fig.2. Bunching Factor, RF Voltage, $\dot{B}$, and the VHF, Time is sec. from T0

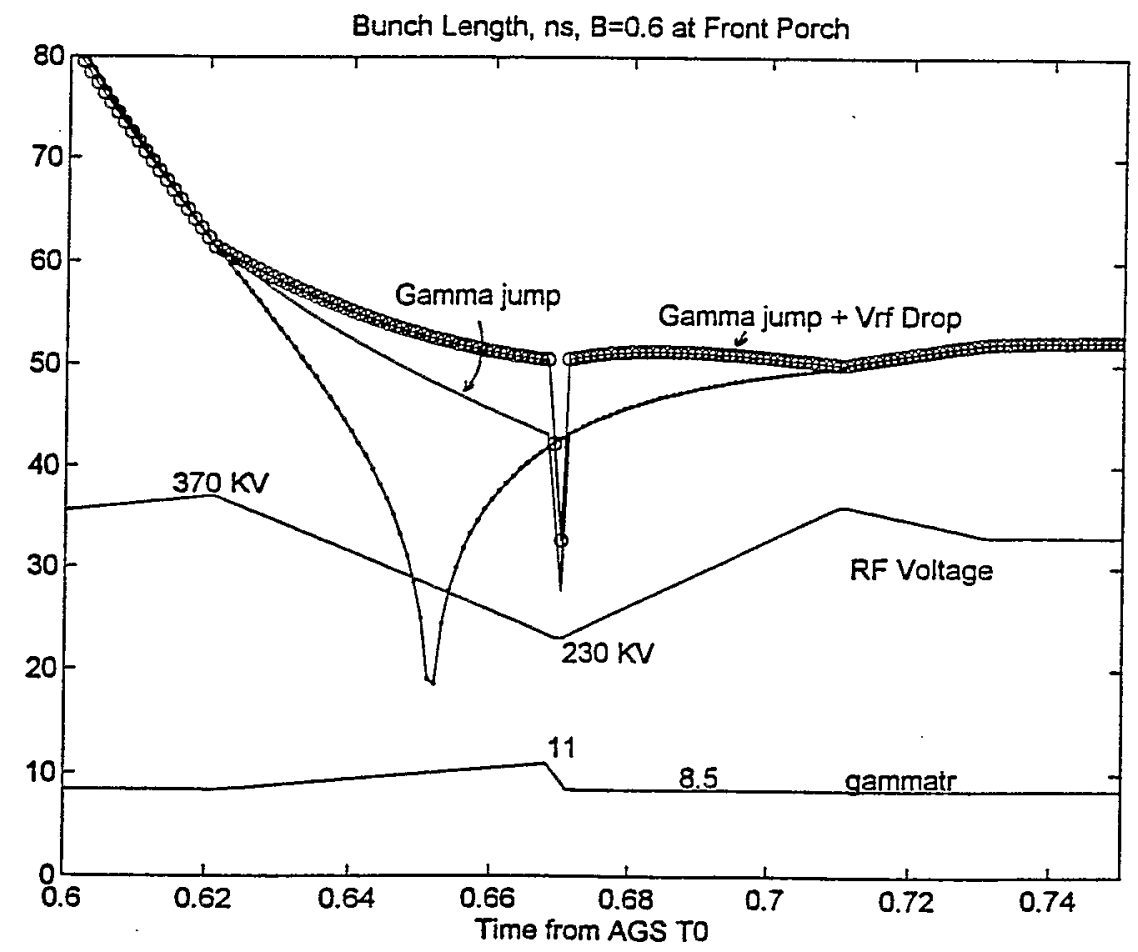

Fig.3. Bunch Length in ns. 


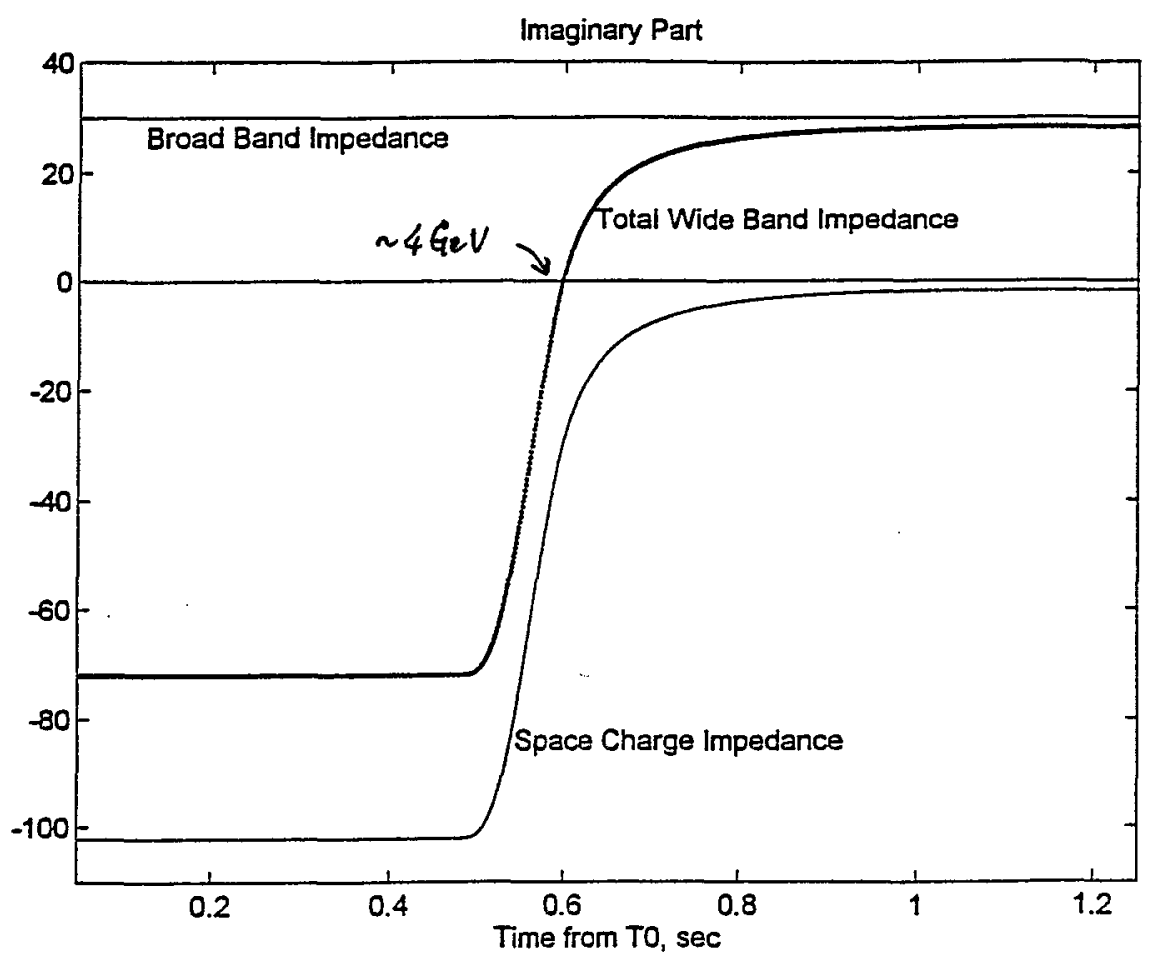

Fig.4. AGS Longitudinal Wide Band Impedance

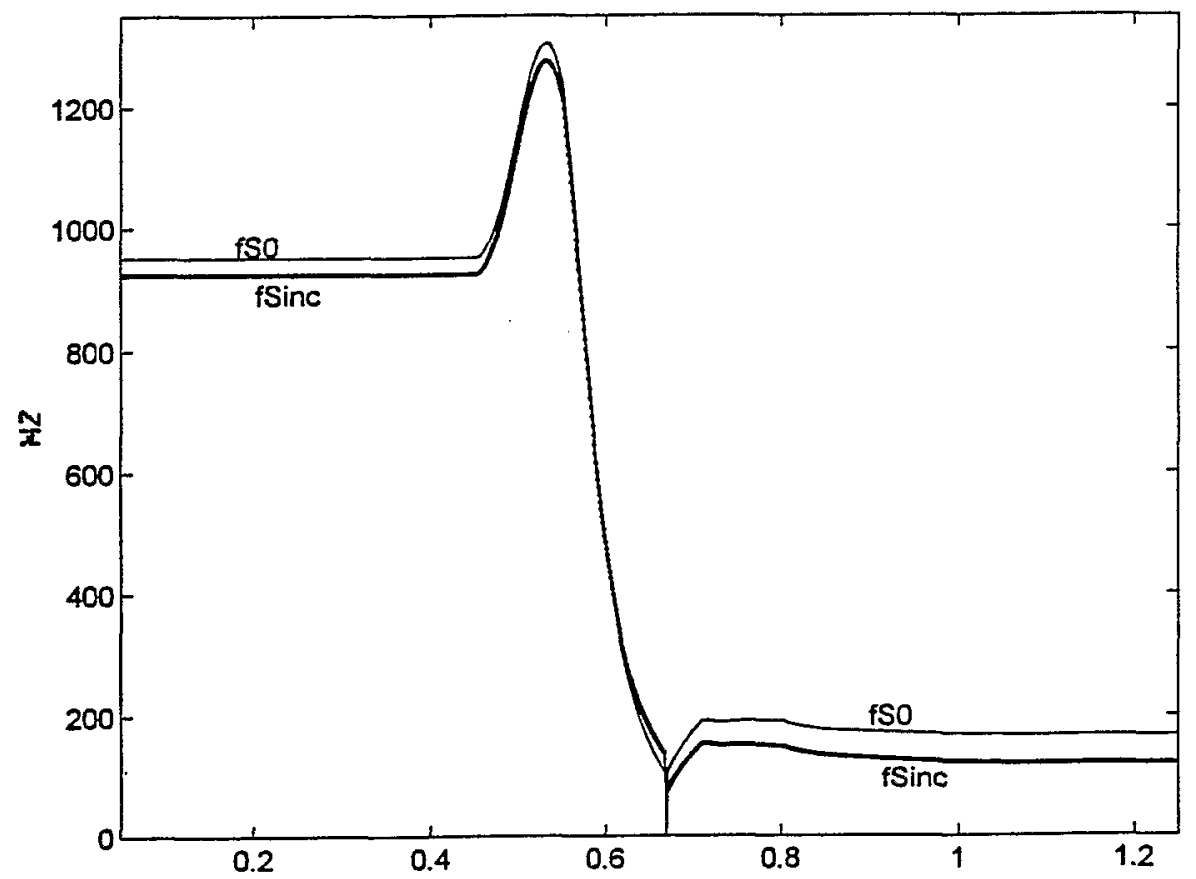

Fig.5. Incoherent Synchrotron Frequency and the One with Zero Intensity 


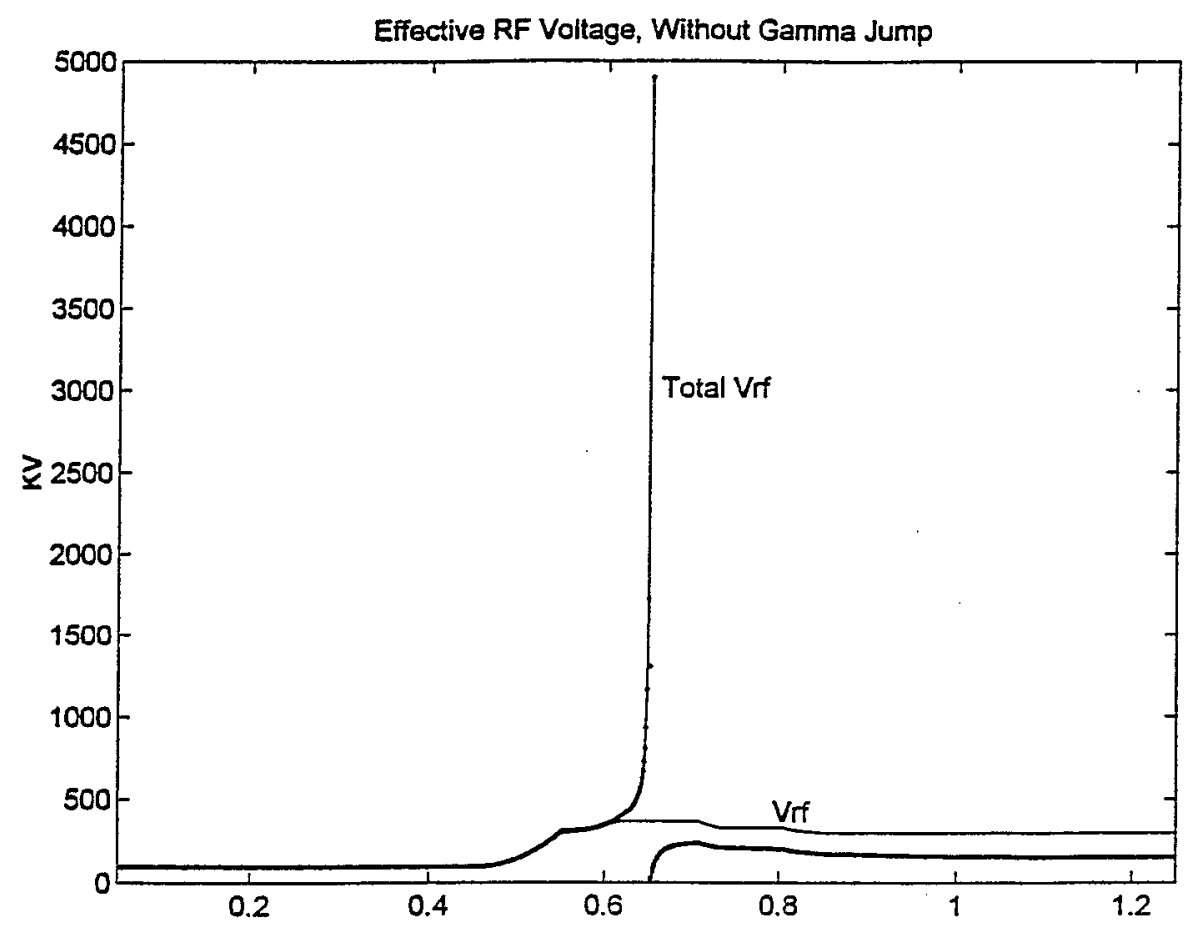

Fig.6. Effective RF Voltage, without Gamma Jump
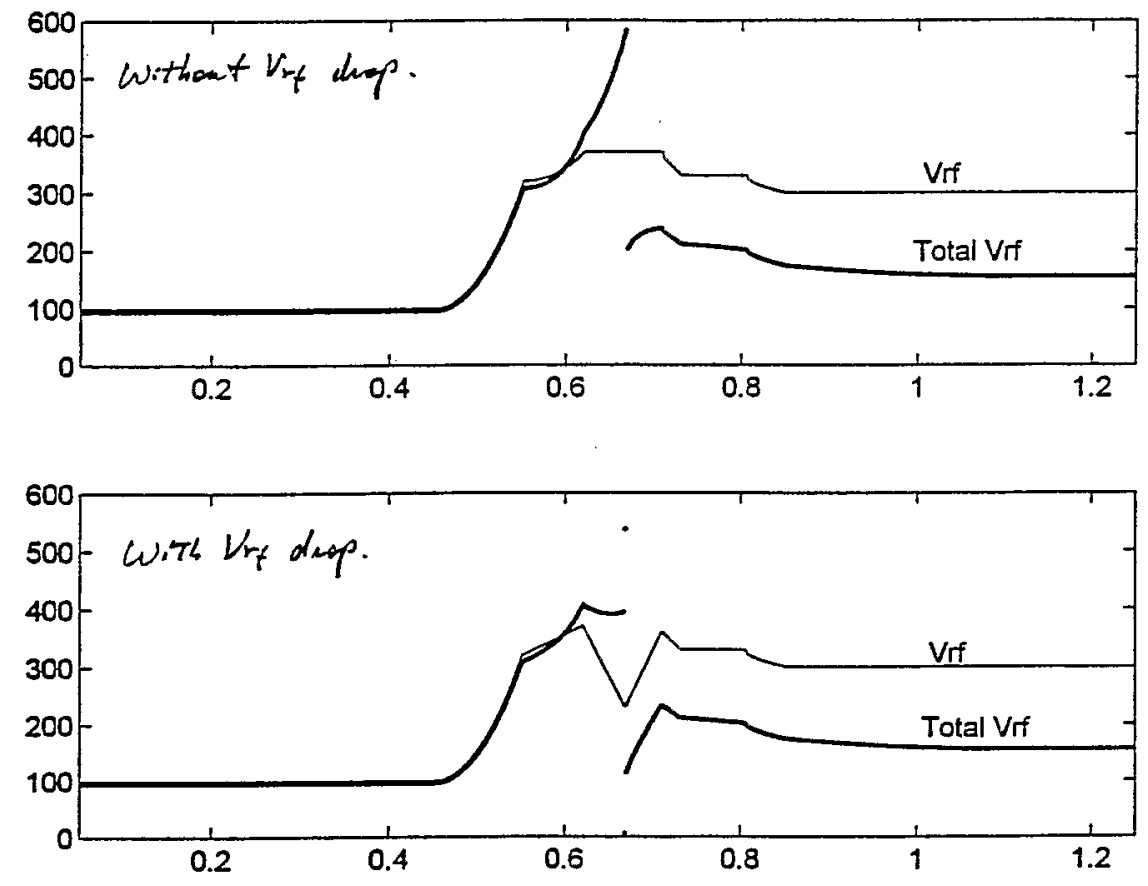

Fig.7. Effective RF Voltage, with Gamma Jump 


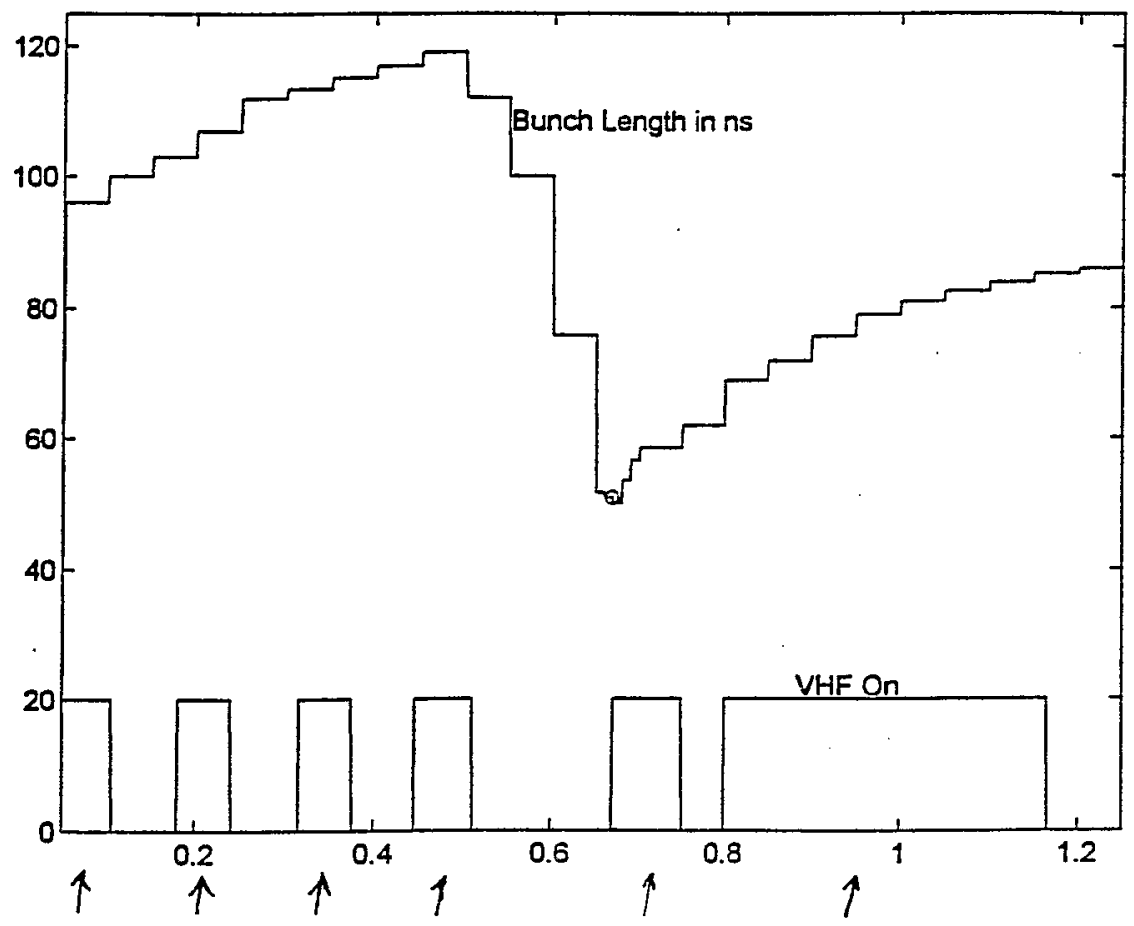

Fig.8. Bunch Length in ns, 1995 SBE

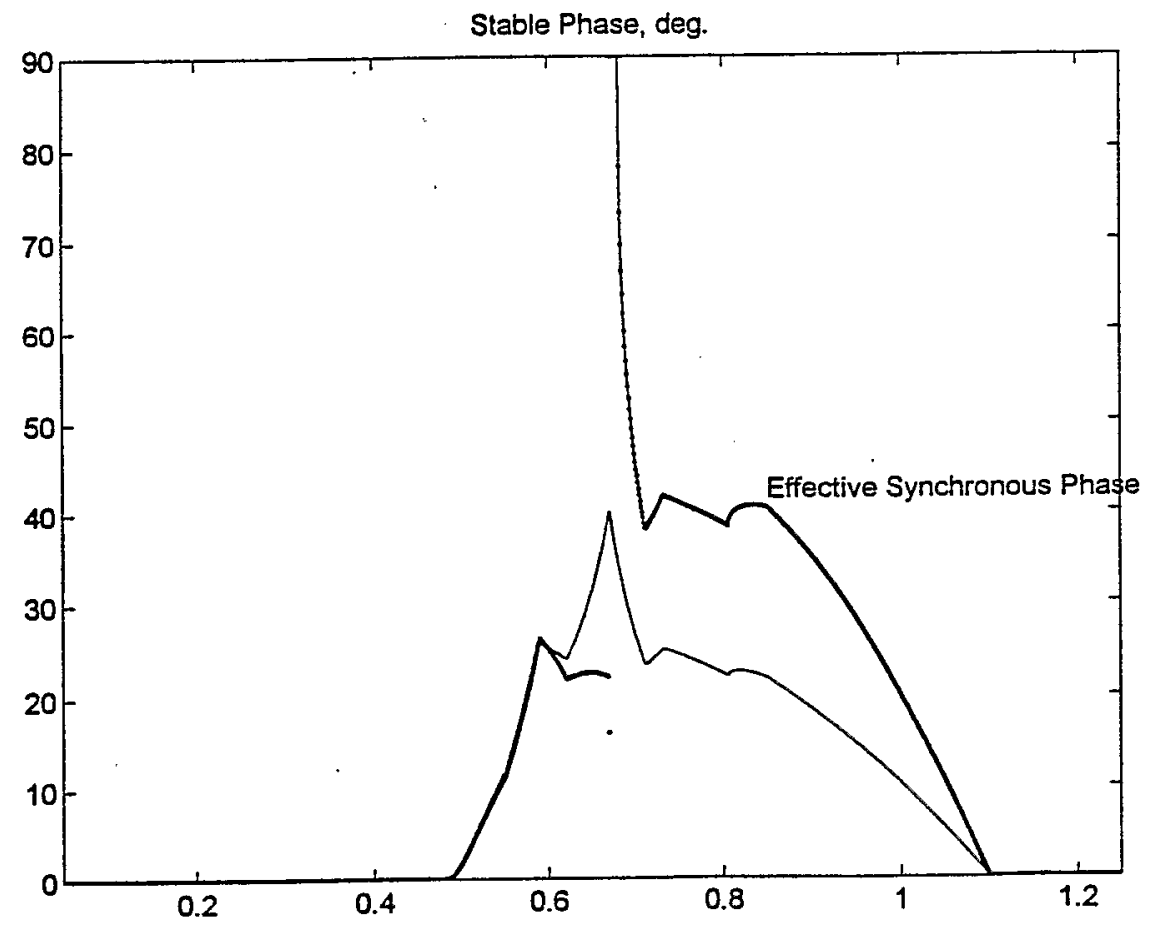

Fig.9. Effective Synchronous Phase, in Degree 


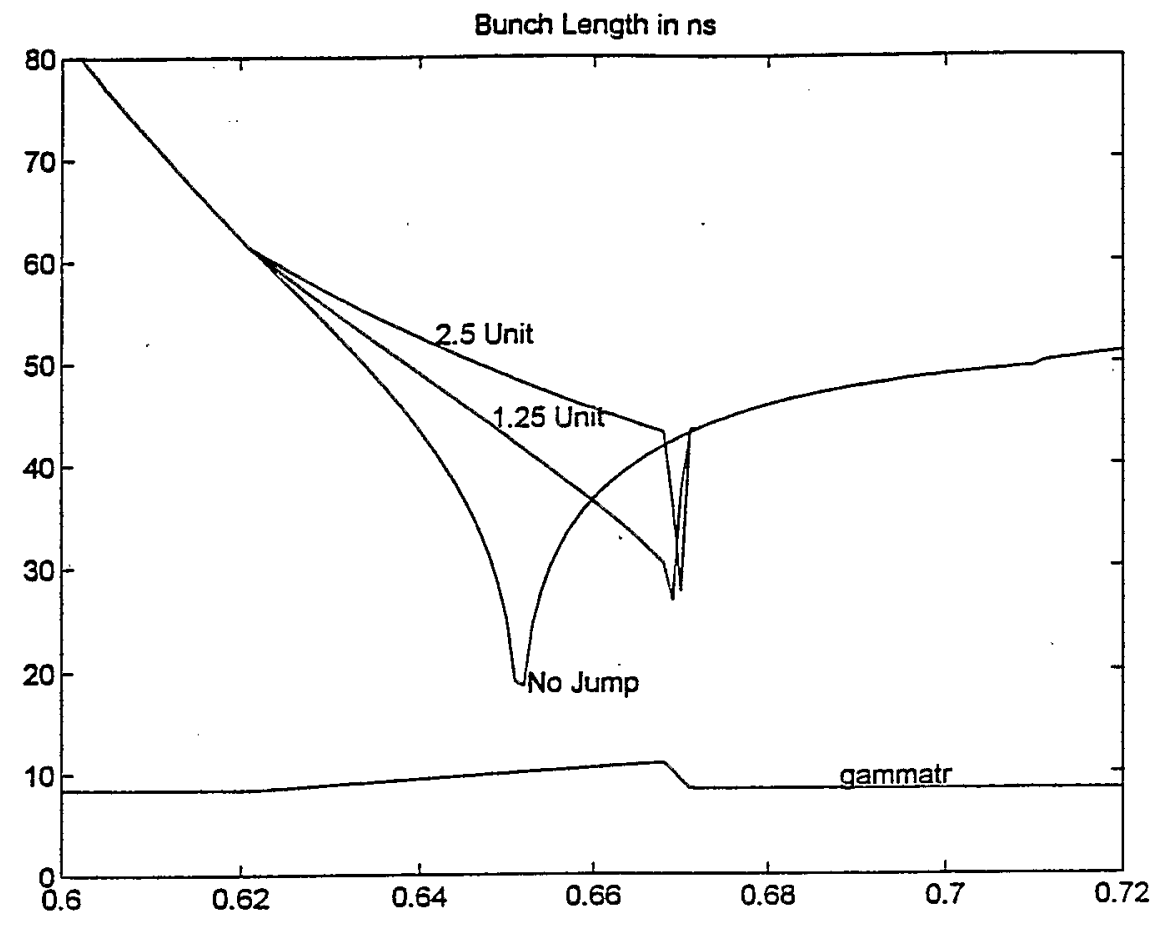

Fig.10. Comparison of Large and Small Gamma Jumps
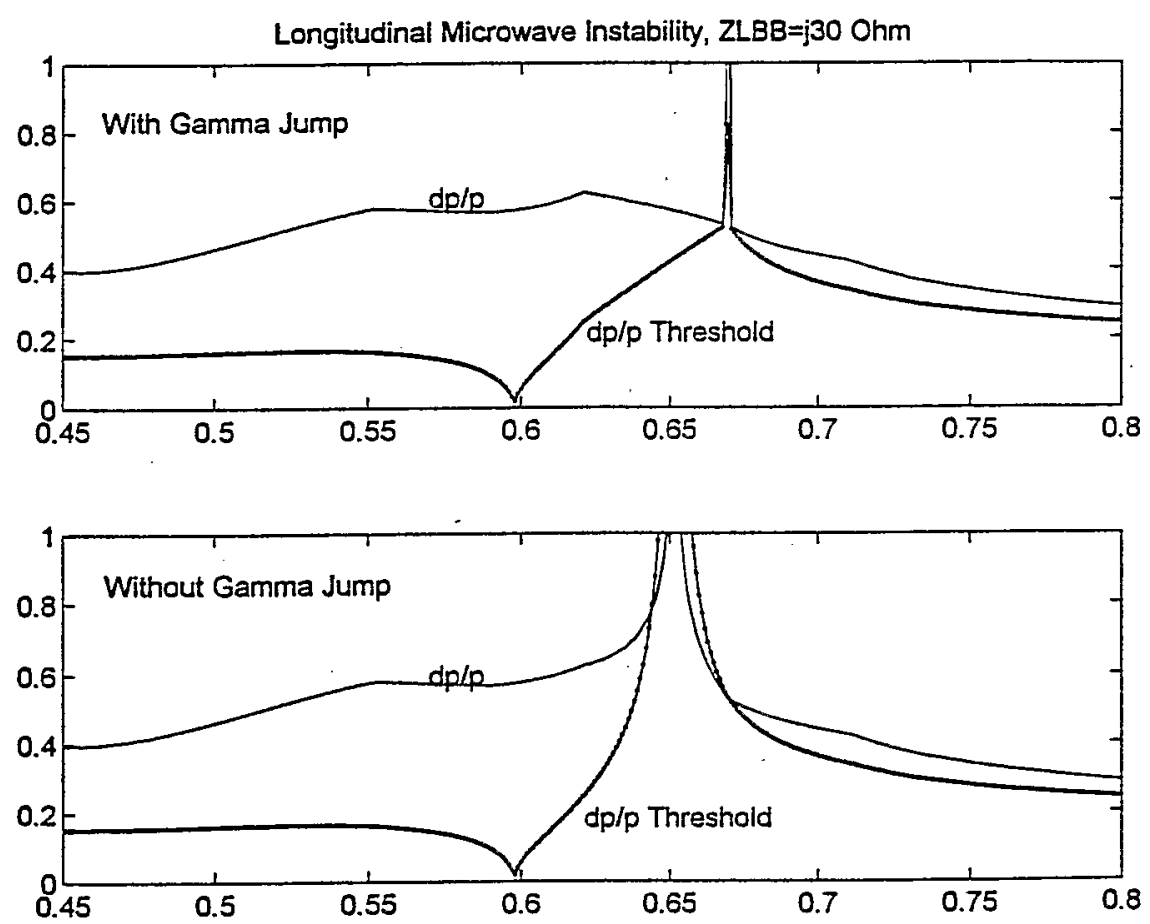

Fig.11. Longitudinal Microwave Instabilities 


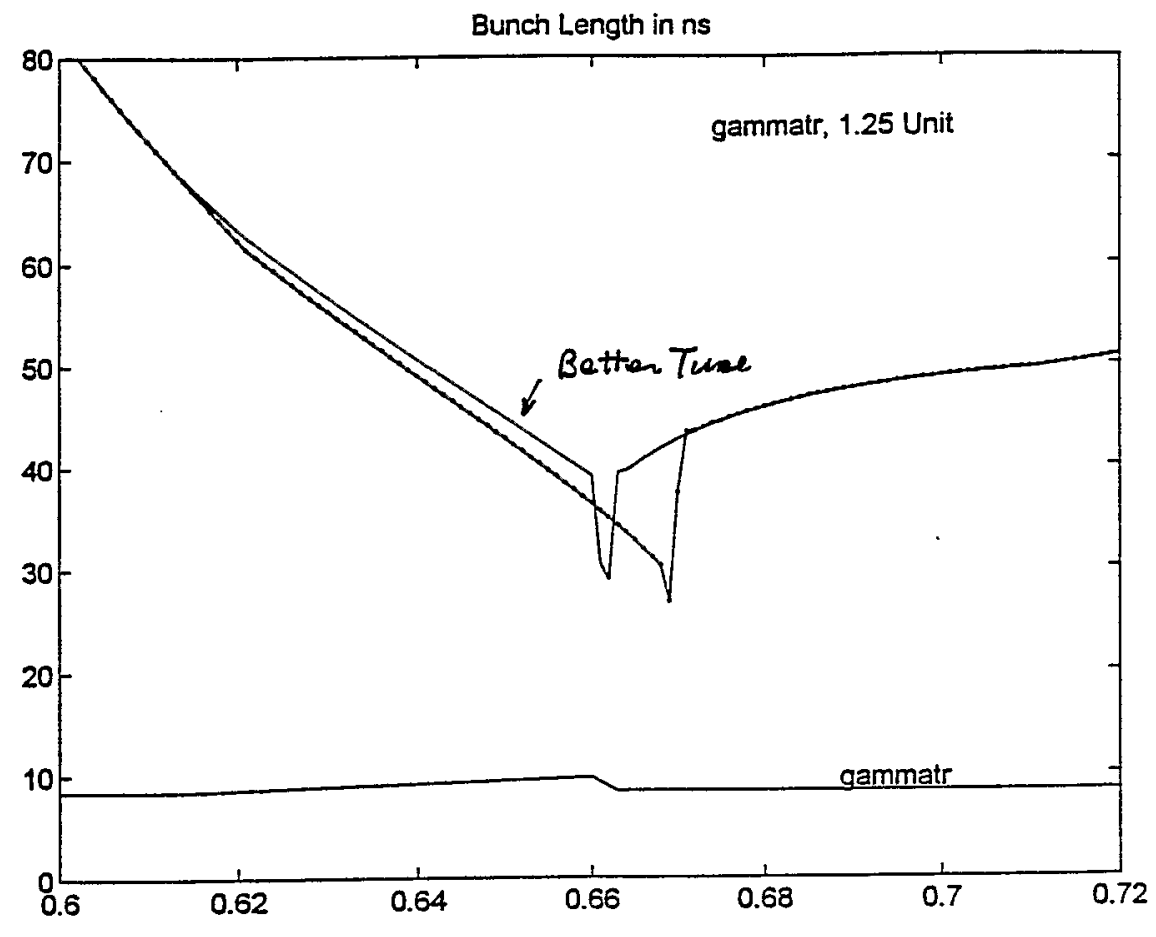

Fig.12. A Better Tune for Small gamma Jump 